\title{
Structural Features Guiding the Design of Liquid-Crystalline Elastomeric Fluorescent Force Sensors
}

\author{
Jaume Garcia-Amorós *(D) and Dolores Velasco *(D) \\ Grup de Materials Orgànics, Institut de Nanociència i Nanotecnologia (IN2UB), Departament de Química \\ Inorgànica i Orgànica (Secció de Química Orgànica), Universitat de Barcelona, Martí i Franquès 1, \\ E-08028 Barcelona, Spain \\ * Correspondence: jgarciaamoros@ub.edu (J.G.-A.); dvelasco@ub.edu (D.V.); \\ Tel.: +34-402-11-97 (J.G.-A.); +34-403-92-60 (D.V.)
}

Received: 23 March 2020; Accepted: 14 April 2020; Published: 16 April 2020

\begin{abstract}
Liquid single crystal elastomers (LSCEs) containing carbazole fluorogenic components alter their luminescence when they are stretched along the director direction. The differential luminescent behavior arises from the distinct interaction between the carbazole fluorophores and their local environment before and after the application of the mechanical input. Indeed, the uniaxial deformation of the material, along its anisotropic direction, forces a closer mesogen-fluorophore interaction, which leads to the quenching of the carbazole luminescence. Importantly, this intermolecular interaction is intimately related to the intrinsic order present in the LSCE. As a result, the amount of light emitted by the material in the form of fluorescence diminishes upon deformation. Thus, the application of mechanical stimuli to liquid-crystalline elastomers furnishes to two interconvertible states for the system with distinct optical properties (with either different emission color or fluorescence intensity). The initial state of the material is completely restored once the applied force is removed. In this way, this kind of macromolecular system can transduce mechanical events into detectable and processable optical signals, thus, having great potential as optical force sensors. In this context, the realization of the distinct structural factors that govern the interactions established between the mesogenic and fluorogenic units at the supramolecular level upon deformation is essential for the development of efficient LSCE-based force sensors. In fact, not only the density of carbazole units and their connection to the main polymer backbone, but also the presence of long range molecular order in the system and the type of mesophase exhibited by the LSCE are key factors for the conception of efficient force sensors based on these self-organized polymer networks. In this review, we present a comprehensive and systematic description of the different features that control the mechanoluminescent behavior of fluorescent liquid-crystalline elastomers and will guide the future design of LSCE-based force sensors with improved performances.
\end{abstract}

Keywords: carbazole; mechanofluorescence; force sensors; liquid-crystalline elastomers

\section{Introduction}

Liquid single crystal elastomers (LSCEs) are weakly crosslinked polymer networks that combine the long range orientational order of liquid crystals, which is uniform along the whole sample, and the elasticity of conventional rubbers [1]. Importantly, the supramolecular organization of the molecules that constitute the liquid-crystalline phase (also known as the mesophase), i.e., the mesogens, can be easily modified through the application of suitable stimuli. As a consequence, liquid-crystalline elastomers are adaptable materials that tune their macroscopic features as a response to specific external inputs [2,3]. For instance, when LSCEs that incorporate azo derivatives within their polymeric network 
are illuminated with light of the appropriate wavelength, the linear trans form of the azo photochrome is transformed into its bent cis counterpart. This photochemical transformation promotes a dramatic disorganization of the surrounding mesogenic molecules and translates into a significant shape change of the material at the macroscopic level. Because of this phenomenon, azobenzene-containing LSCEs find application as light-driven artificial actuators (i.e., materials capable of converting light into mechanical actions) [4-10].

LSCEs can also be engineered to perform exactly the opposite task, that is, to encode mechanical inputs into optical signals. For this purpose, the intermolecular interactions established between the mesogenic molecules and rationally designed luminescent labels must be altered upon the application or release of a mechanical stimulus [11-18]. In this way, the introduction of selected organic fluorophores into this type of self-organized polymeric system enables the modulation of the amount of light emitted in the form of fluorescence upon deformation, giving rise to LSCE-based optical force sensors [19]. To the best of our knowledge, only two examples of LSCE-based optical force sensors have been reported in addition to the ones described herein. In these instances, the distinct emission intensity, detected when the elastomeric material undergoes its nematic-to-isotropic phase transition, was exploited as the driving force of the sensor $[20,21]$. These materials require, however, relatively high temperatures to operate. During recent decades, non-liquid-crystalline polymers and elastomers have also been exploited as excellent matrixes to perform mechanochromic reactions for force sensing purposes. In these cases, mechanophoric components, typically spiropyranes and their derivatives, are covalently attached to a polymer backbone. In the relaxed state, the mechanophore is in its spiro closed form and the material is completely colorless. However, when the mechanical stimulus is applied, the opened merocyanine form of the mechanophore is generated and the material becomes colored, thus enabling the visualization of the applied force to the naked eye [22-24]. Other mechanophores, such as diarylbibenzofuranones [25] and bis(adamantyl)-1,2-dioxetane bisacrylate [26], have also been explored for this purpose. Force sensing materials have also been achieved through the covalent attachment of mechanoresponsive organic fluorophores to non-liquid-crystalline polymers. In some of these examples, the sensing abilities of the material come from the fact that the organic fluorophore changes its aggregation state upon mechanical stimulation, thereby generating two distinct emissive states [27-29]. Supramolecular chemistry has also been exploited recently to modulate the emissive properties of non-liquid-crystalline polymers and elastomers [30,31]. All these mechanosensitive networks find great application in actual technology as damage detectors. It is also interesting to stress at this point that mechanochemical reactions can also be performed in polymeric matrixes containing carefully chosen mechanophores with an extremely high spatial precision, permitting 3D nanoprinting and the production of nanodevices and nanosensors for measuring mechanical stress in situ [32]. It should be also mentioned that non-polymeric materials, mainly crystalline solids, have also been proved to change their luminescence upon grinding, smearing, shearing, etc., due to drastic modifications of their crystalline structures [33-51]. However, in many of these instances, the initial state of the system must be recovered either by recrystallization or by exposing the solid to vapors of a suitable solvent. This fact increases considerably the response time of the material and compromises its reversibility, thereby limiting its potential applicability.

Elastic materials with the ability to detect small deformations, forces or pressures are of great importance within a wide variety of areas, ranging from information transmission technology to biomedicine. In this scenario, mechanofluorescent LSCEs emerge as valuable candidates. Thus, gaining insight into the mechanisms underlying mechanofluorescence in LSCEs is essential to pave the way to a new generation of mechanoluminescent elastomeric materials with novel functionalities and enhanced performances. In this context, a careful design of the carbazole fluorophores at the molecular level and a proper engineering of the intermolecular interactions established with the surrounding mesogenic molecules is fundamental to improve the efficiency of the resulting force sensor. Our research group has been involved during recent years in the development of carbazole-containing LSCEs for efficient optical force sensing. In particular, we have explored the impact of not only several features concerning 
the carbazole fluorophores but also the presence of a long range orientational and positional order in the LSCE on the sensing abilities of the system. Therefore, it is the main aim of this review to present the reader the main progresses achieved on the way to efficient optical force sensors based on fluorescent liquid single crystal elastomers. Specifically, five main features have been investigated so far, which are illustrated schematically in Scheme 1 and summarized below:

- Fluorophore content.

- Presence of long range orientational order in the host matrix.

- Length of the flexible spacer connecting the carbazole fluorophores to the polymer backbone.

- Anchoring mode (side-on or end-on) of the fluorogenic moieties.

- Type of mesophase exhibited by the elastomeric material.
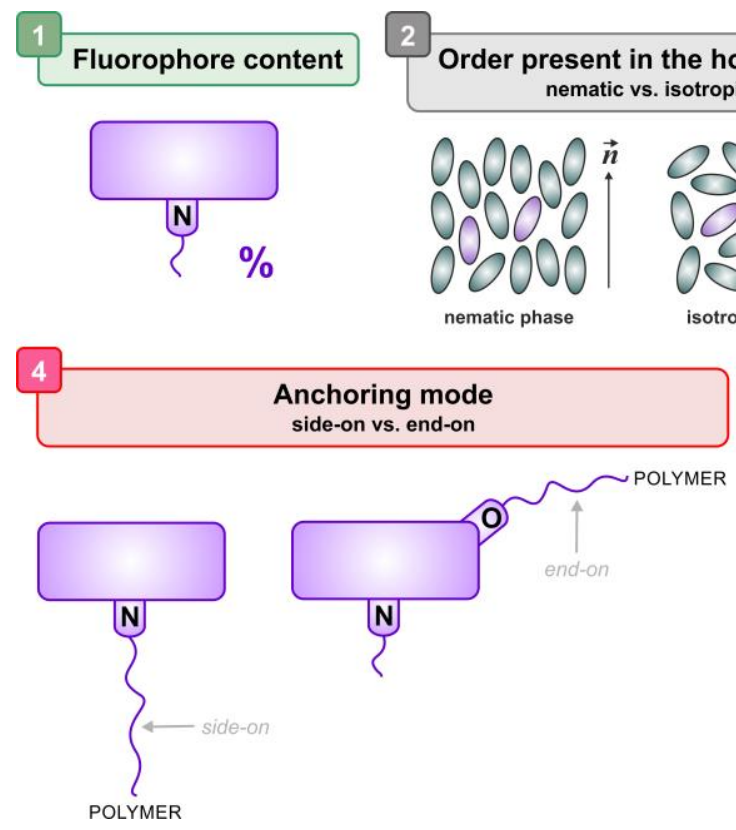

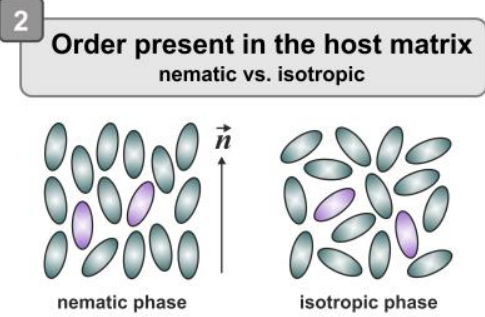

isotropic phase
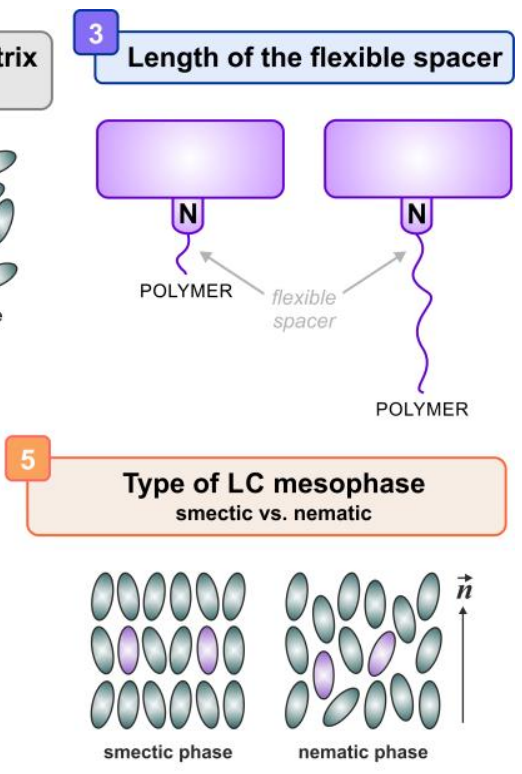

Scheme 1. Schematic representation of the different features that govern the mechanoluminescent behavior of LSCE-based optical force sensors.

\section{Discussion}

\subsection{Design, Synthesis and Characterization of the Mechanofluorescent Elastomers}

This study will deal with the mechanofluorescent behavior of nematic and smectic LSCEs (smectic LSCEs will be described in detail in Section 2.2.5, vide infra). The different elastomers will be denoted as follows: EMm_CBZX $n \_Z$, where M $m$ indicates the mesogenic monomer (M4 or M10) used, $\mathrm{X} n$ states the atom ( $\mathrm{N}$ or $\mathrm{O}$ ) and the length of the flexible spacer through which the carbazole fluorophore is attached to the elastomeric network, and $\mathrm{z}$ informs about the carbazole content (see Scheme 2). All nematic LSCEs are composed of a main polymer backbone of polyhydrogeno-methylsiloxane (PHMS in Scheme 2) functionalized with the nematic mesogen 4-methoxyphenyl 4-(3-butenyloxy)benzoate (M4 in Scheme 2). The use of this organic molecule in high proportions (70\%-85\% mol) endows the resulting elastomer with a stable nematic mesophase over a wide range of temperatures. In addition, 1,4-di-(10-undecenyloxy)benzene (CL in Scheme 2) is used as a cross-linking agent ( $10 \% \mathrm{~mol})$ to connect the distinct polymeric chains and confer the required elastic properties to the final material. Finally, as we have already mentioned, it is our main aim to transduce mechanical forces into optical signals. For this task, carbazole derivatives (CBZX $n$ in Scheme 2) have been introduced as fluorogenic components in the elastomeric network at small concentrations (from $5 \%$ to $20 \% \mathrm{~mol}$ ). Carbazole, 
a well-known organic emitter, has been selected as a fluorophore not only due to its high fluorescence quantum yield and stability, but also because it can be easily functionalized at the required positions by means of well-established procedures.
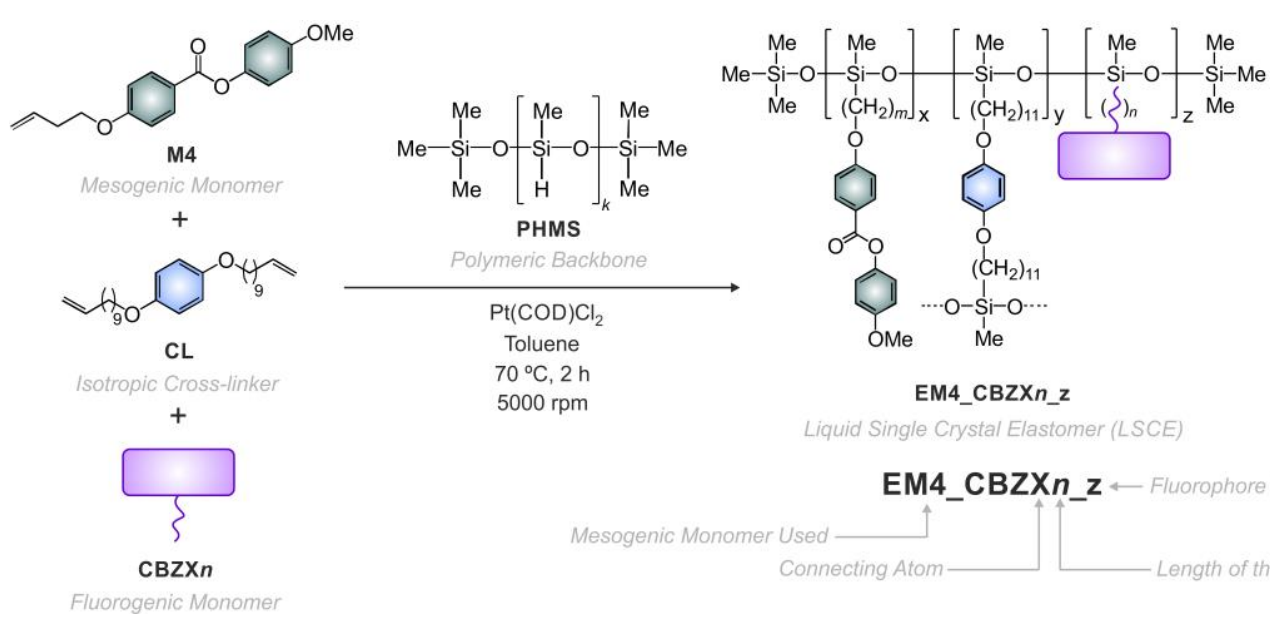

EM4_CBZXn_z F Fluorophore Content

Scheme 2. Chemical structure and composition of the nematic liquid single crystal elastomers EM4_CBZXn_z.

LSCEs have been synthesized according to the procedure described by Küpfer and Finkelmann in 1991 [52]. According to this methodology, LSCEs can be prepared via a three-step protocol. In the first step, a partial Pt-catalyzed hydrosilylation reaction is carried out, where the distinct monomers react with the PHMS backbone through their terminal olefins. It should be highlighted that this reaction is stopped at its early stages (only after $2 \mathrm{~h}$ at $70{ }^{\circ} \mathrm{C}$ ), since the main aim of this part of the process is to obtain a stable but tunable network. In the second step, the elastomeric sample is stretched along its longest axis in order to align the different nematic directors. After the second step, a monodomain liquid-crystalline elastomer, i.e., a LSCE, is obtained. In the third and final step, the sample is left to react at $70{ }^{\circ} \mathrm{C}$ for 2 days, without removing the applied force, to fix the anisotropy generated previously. Afterwards, the non-reacted monomers and the catalyst are removed from the material by a swelling-deswelling procedure, using toluene and hexane, respectively. A detailed description of the synthetic procedure can be found in [53].

The mesophase structure and the mesomorphic behavior of the prepared samples were analyzed by X-Ray diffraction (XRD) and differential scanning calorimetry (DSC). XRD was used to establish unambiguously the type of liquid-crystalline phase exhibited by the LSCE. Nematic liquid-crystalline elastomers reveal a broad reflex in the wide-angle region of their X-ray scattering pattern $\left(2 \theta \approx 20^{\circ}-21^{\circ}\right)$. From this reflex, the mesogens spacing is calculated by applying Bragg's law. On the other hand, the presence of a series of additional reflexes in the small-angle region of the $\mathrm{X}$-ray scattering pattern is indicative of a smectic-like organization. In this case, the mesogens and layers spacing is calculated from the wide-angle and the first-order small-angle reflex, respectively. Furthermore, the azimuthal intensity maxima for the first-order small-angle reflex, located at $0^{\circ}$ and $180^{\circ}$, evidence that the mesogens are not tilted and their longest axis is parallel to the layer normal. It should be also mentioned that the presence of a single and sharp wide-angle reflex at $2 \theta \approx 20^{\circ}-21^{\circ}$ together with the absence of additional sharp reflections in the wide-angle region points out the existence of a hexatic smectic $B$ mesophase. At higher temperatures, the broadening of this reflex reflects the transformation of the hexatic smectic B phase into a smectic A mesophase.

The order parameter $\mathrm{S}$ for the average orientation of the long molecular axis of the mesogenic side groups with respect to the main axis of the LSCE is measured from the azimuthal dependence of the intensity at a scattering vector corresponding to the maximum of the wide-angle reflex $(2 \theta \approx$ 
$\left.20^{\circ}-21^{\circ}\right)$. The value of the order parameter is determined by the method reported by Mitchell and Lovell $[54,55]$. In all the cases, the highly anisotropic azimuthal intensity distribution of this reflex, showing intensity maxima at $90^{\circ}$ and $270^{\circ}$, indicates that the mesogens are homogeneously organized all over the elastomer, i.e., that LSCEs have been obtained.

The thermal range of stability of the liquid-crystalline mesophase/s is assessed by differential scanning calorimetry. DSC thermograms reveal that the system adopts an enantiotropic liquid-crystalline mesophase (nematic or smectic B) between a glass transition temperature $\left(T_{\mathrm{g}}\right)$ and a liquid crystal-to-isotropic phase transition, with the exception of the smectic elastomers, where a smectic B-to-smectic A phase transition was also detected before the isotropization of the LSCE. All LSCEs exhibit a liquid-crystalline nature under ambient conditions.

The mechanofluorescent behavior of the different elastomers, which is directly related with their sensing abilities, was evaluated from the variation observed in the LSCE luminescence upon deformation (in Figure 1a) at room temperature. These analyses were carried out by registering the emission spectrum of the elastomeric material before and after applying a uniaxial deformation $(\varepsilon$ in Figure 1) along the sample longest axis (coincident with $n$ ) in a stepped fashion (in Figure 1b). Excitation of the carbazole fluorophores at $\lambda_{\mathrm{Ex}}=290-315 \mathrm{~nm}$ results in a strong emission within the UV region of the electromagnetic spectrum. Mechanofluorescence experiments imply the monitoring of the amount of light emitted by the LSCE in the form of fluorescence at the wavelength of maximum emission $\left(\lambda_{\mathrm{Em}}=350-360 \mathrm{~nm}\right)$ while it is stretched along the anisotropic direction of the sample. As a whole, fluorescence intensity, $I_{\mathrm{F}}$ (or in relative terms, $\Delta I_{\varepsilon}=\left(I_{\varepsilon}-I_{0} / I_{0}\right) \times 100$, where $I_{\varepsilon}$ and $I_{0}$ correspond to the fluorescence intensity of the stretched and unstretched sample, respectively) drops dramatically upon deformation (in Figure $1 b, c)$ until a plateau is eventually reached. This constant value ( $\Delta I_{\text {Max }}$ in Figure 1), which quantifies the force sensor efficiency, corresponds to the maximum variation in the material luminescence that can be induced upon mechanical stimulation and it is the benchmarking criterion of the performance of optical force sensors.
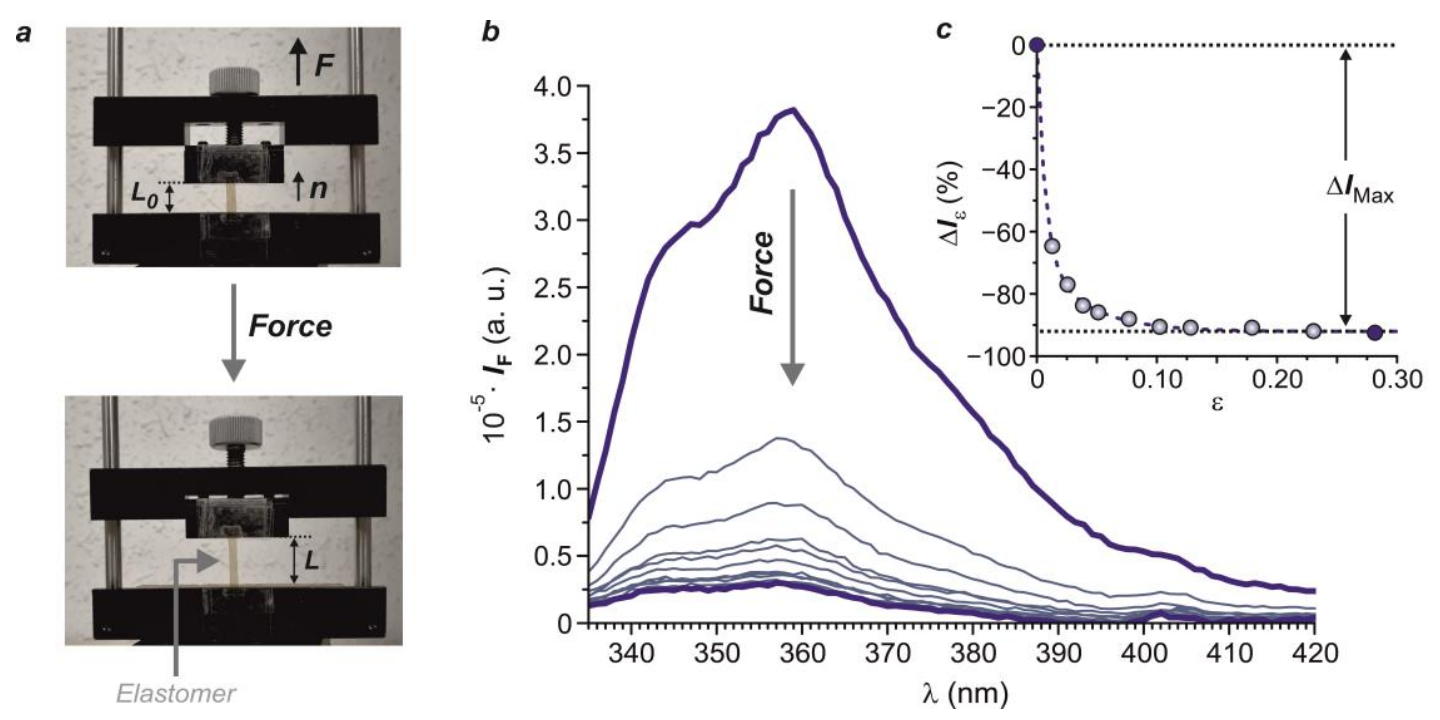

Figure 1. (a) Deformation of an LSCE along the direction director, (b) evolution of the emission spectrum, and (c) variation of the relative emission intensity, $\Delta I_{\varepsilon}$, of a representative LSCE upon applying a uniaxial deformation $\varepsilon$ along its anisotropic direction.

\subsection{Structural Factors That Impact the Mechanofluorescent Behavior}

\subsubsection{Fluorophore Content in the Elastomeric Network}

One of the parameters that influences the mechanoluminescence exhibited by carbazole-based LSCEs is the concentration of the organic fluorophore incorporated into the elastomeric network [19]. 
In order to illustrate this point, three different elastomers will be initially considered, which contain increasing amounts of 9-(5-hexenyl)-9H-carbazole as the fluorogenic component. Specifically, this molecule was introduced in a 5\%,10\% and 20\% mol, yielding LSCEs EM4_CBZN6_5, EM4_CBZN6_10 and EM4_CBZN6_20 (in Figure 2a), respectively. The XRD pattern (in Figure 2b) for elastomers EM4_CBZN6_5 and EM4_CBZN6_10 at $298 \mathrm{~K}$ displays two equatorial reflexes in the wide angle X-ray scattering regime (mesogens spacing $\approx 4.4 \AA$ ), evidencing a macroscopically aligned sample (order parameter $=0.74$ ) with a nematic liquid-crystalline phase under ambient conditions. DSC thermograms reveal that the nematic mesophase is stable between the glass transition temperature at $T_{\mathrm{g}}=276 \mathrm{~K}$ and the nematic-to-isotropic phase transition temperature, $T_{\mathrm{N}-\mathrm{I}}$, at 327 and $313 \mathrm{~K}$, respectively. The mesogenic and mechanofluorescent behavior of elastomer EM4_CBZN6_20 will be described in detail in Section 2.2.2 due to its isotropic nature.
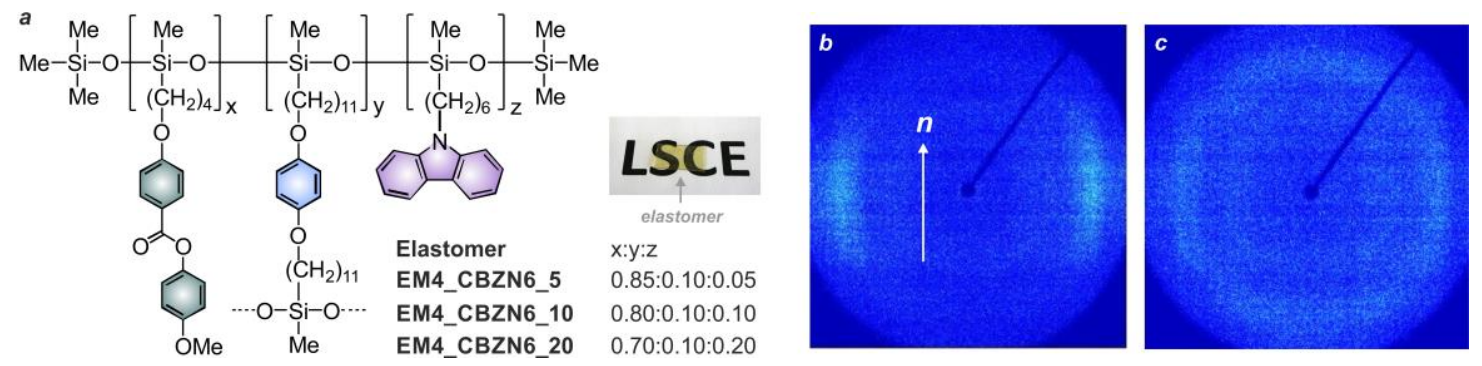

Figure 2. (a) Chemical structure of elastomers EM4_CBZN6_5, EM4_CBZN6_10 and EM4_CBZN6_20 and the X-ray diffraction pattern for elastomers EM4_CBZN6_10 (b) and EM4_CBZN6_20 (c).

Elastomer EM4_CBZN6_5, which contains a 5\% mol of carbazole units, exhibits a $\Delta I_{\text {Max }}$ value of $-27 \%$ (Figure 3). On the other hand, LSCEs EM4_CBZN6_10, which contains a two-fold higher concentration of carbazole fluorophores shows an increased efficiency of $-34 \%$ [19]. In this way, a higher carbazole content within the elastomeric network leads to more efficient optical force sensors.
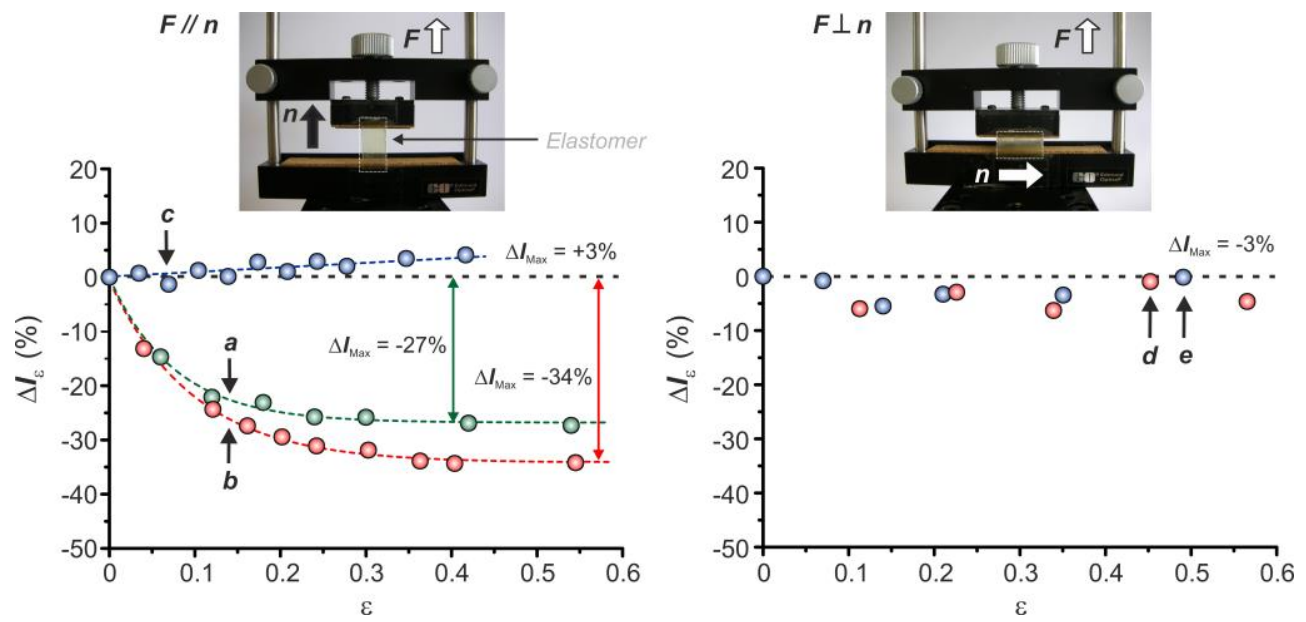

Figure 3. Mechanofluorescent response for elastomers EM4_CBZN6_5 (a), EM4_CBZN6_10 (b,d) and EM4_CBZN6_20 (c,e) upon deformation along the direction parallel $(\mathbf{a}-\mathbf{c})$ and perpendicular $(\mathbf{d}, \mathbf{e})$ to the longest axis of the elastomeric sample [19]. (Copyright from Wiley-VCH Verlag GmbH \& Co. KGaA 2015).

\subsubsection{Presence of Long Range Orientational Order in the Host Matrix}

While LSCEs EM4_CBZN6_5 and EM4_CBZN6_10 exhibit a stable nematic mesophase over a wide range of temperatures (from 276 to 327 and $313 \mathrm{~K}$, respectively; see above), elastomer EM4_CBZN6_20, 
with the highest carbazole content, presents uniquely a glass transition at $T_{\mathrm{g}}=269 \mathrm{~K}$ being, thus, isotropic at room temperature. Accordingly, the corresponding X-ray diffraction pattern (in Figure 2c) shows only a diffused halo under these experimental conditions. Remarkably, when mechanofluorescence experiments are carried out with this elastomer, no variation in the emission intensity is detected upon deformation along its longest axis $\left(\Delta I_{\operatorname{Max}}=+3 \%\right.$, in Figure 3c) [19]. The differential behavior observed between the isotropic elastomer EM4_CBZN6_20 and its nematic analogs EM4_CBZN6_5 and EM4_CBZN6_10 is explained in terms of the presence of long range orientational order in this spatial direction for the latter elastomers. When the elastomeric sample is stretched parallel to $n$, nematic order increases with a concomitant stronger interaction between the mesogenic units [56]. Similarly, the deformation of the elastomeric material also results in a more favorable carbazole-mesogen interaction. This fact induces a fluorescence quenching of the carbazole moieties and provokes, in turn, the decrease in fluorescence intensity observed at the macroscopic level. Thus, the mechanoluminescent properties displayed by these elastomeric materials are coupled directly with the intrinsic liquid crystal order of the system.

This statement has also been corroborated by means of mechanofluorescence experiments performed along the shortest axis of the sample. When either nematic (EM4_CBZN6_5 and EM4_CBZN6_10) or isotropic (EM4_CBZN6_20) elastomers are deformed along the direction where neither orientational nor positional order exists, only a residual mechanoluminescence is detected $\left(\Delta I_{\operatorname{Max}}=-3 \%\right.$, in Figure $\left.3 \mathrm{~d}, \mathrm{e}\right)$. Therefore, this observation highlights, once again, that an elastomeric material with an intrinsic liquid-crystalline nature is essential to conceive optical force sensors based on these self-organized macromolecular networks [19].

\subsubsection{Length of the Fluorophore Flexible Spacer}

Another structural feature that impacts the efficiency of LSCE-based optical force sensors is the length of the fluorophore flexible spacer, i.e., the length of the alkyl chain that connects the carbazole moieties to the main polymer backbone [57]. The role of this particular constitutional factor will be illustrated by considering the four different elastomers: EM4_CBZN2_10, EM4_CBZN3_10, EM4_CBZ N6_10 and EM4_CBZN11_10 (in Figure 4a), where the length of the flexible spacer of the carbazole monomers increases gradually from sample to sample. These four elastomers also exhibit a monodomain nematic mesophase, which is stable between the glass transition temperature at 276-282 K and the nematic-to-isotropic phase transition temperature at 305-336 K.
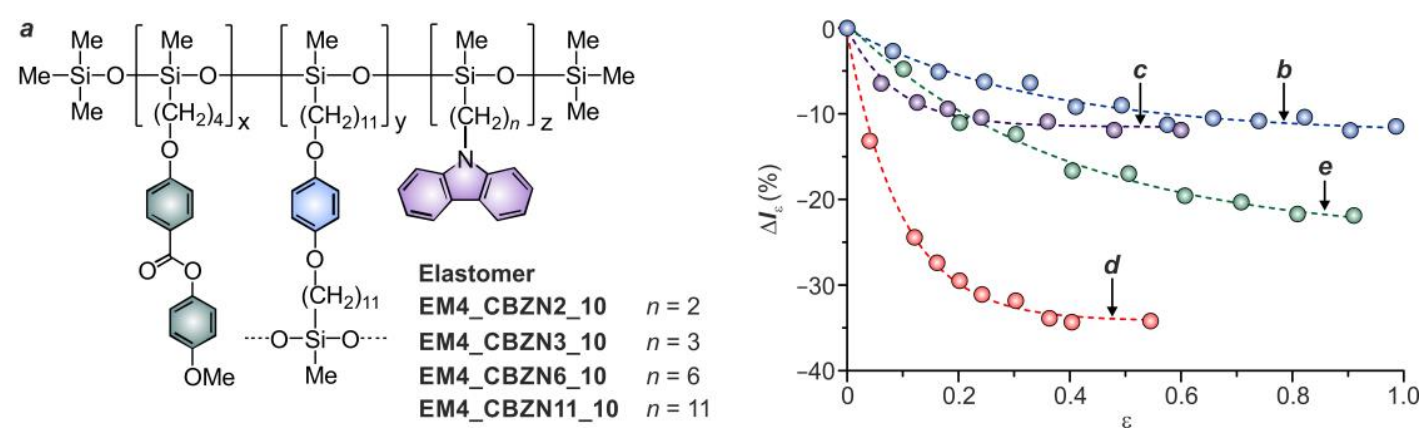

Figure 4. Chemical composition (a) and mechanofluorescent response for LSCEs EM4_CBZN2_10 (b), EM4_CBZN3_10 (c), EM4_CBZN6_10 (d) and EM4_CBZN11_10 (e) [57]. (Copyright from PCCP Owner Societies 2016).

$\Delta I_{\text {Max }}$ depends significantly on the length of the flexible spacer that links the pendant carbazole fluorophores to the elastomeric network. On the one hand, when the fluorogenic components are attached to the polymer through either the shortest (EM4_CBZN2_10 and EM4_CBZN3_10) or the longest (EM4_CBZN11_10) spacers, the elastomeric material suffers the smallest decrease $\left(\Delta I_{\text {Max }}\right.$ of $-12 \%,-11 \%$ and $-22 \%$, respectively; in Figure $4 \mathrm{~b}-\mathrm{e}$ ) of its fluorescence intensity upon deformation. 
On the other hand, LSCE EM4_CBZN6_10, where the carbazole fluorophores have a flexible spacer of a similar length to that of the mesogen M4, exhibits the greatest mechanoluminescent response $\left(\Delta I_{\operatorname{Max}}=-34 \%\right.$, in Figure $\left.4 \mathrm{~d}\right)$ [57]. This latter result has been ascribed to the more favorable overlap between the carbazole units and the surrounding mesogen molecules that occurs when they bear flexible spacers of a comparable length. Indeed, short spacers do not permit an efficient overlap between their rigid cores. In addition, the very high conformational freedom displayed by long spacers enables a moderate interaction between carbazole and M4 molecules, but it does not allow an efficient transmission of the external mechanical input to the fluorogenic components. In both instances, less effective optical force sensors are obtained. Therefore, the length of the fluorophore flexible spacer controls not only the transmission of the external mechanical input from the main polymer backbone to the mesogenic and fluorogenic moieties, but also the interaction between them both, which determines the efficiency of the final optical force sensor.

\subsubsection{Anchoring Mode (End-on Versus Side-on)}

To show the effect of the anchoring mode, i.e., end-on or side-on, two nematic liquid single crystal elastomers will be considered: EM4_CBZO6_10 and EM4_CBZN6_OMe_10 (in Figure 5a,b) [58]. The former elastomer has its carbazole fluorophores connected by a lateral alkoxyl chain in an end-on fashion. The latter, which resembles elastomer EM4_CBZN6_10 (see Figure 2), is connected to the main polymer backbone by the nitrogen atom of the heterocycle in a side-on mode. It has to be noticed that the fluorophores of LSCE EM4_CBZN6_OMe_10 contain a methoxy group in one of the meta positions of the carbazole ring in order to have identical transition moments for both elastomeric systems. DRX and DSC experiments confirm that the two elastomers exhibit a monodomain nematic mesophase, which is stable under ambient conditions.
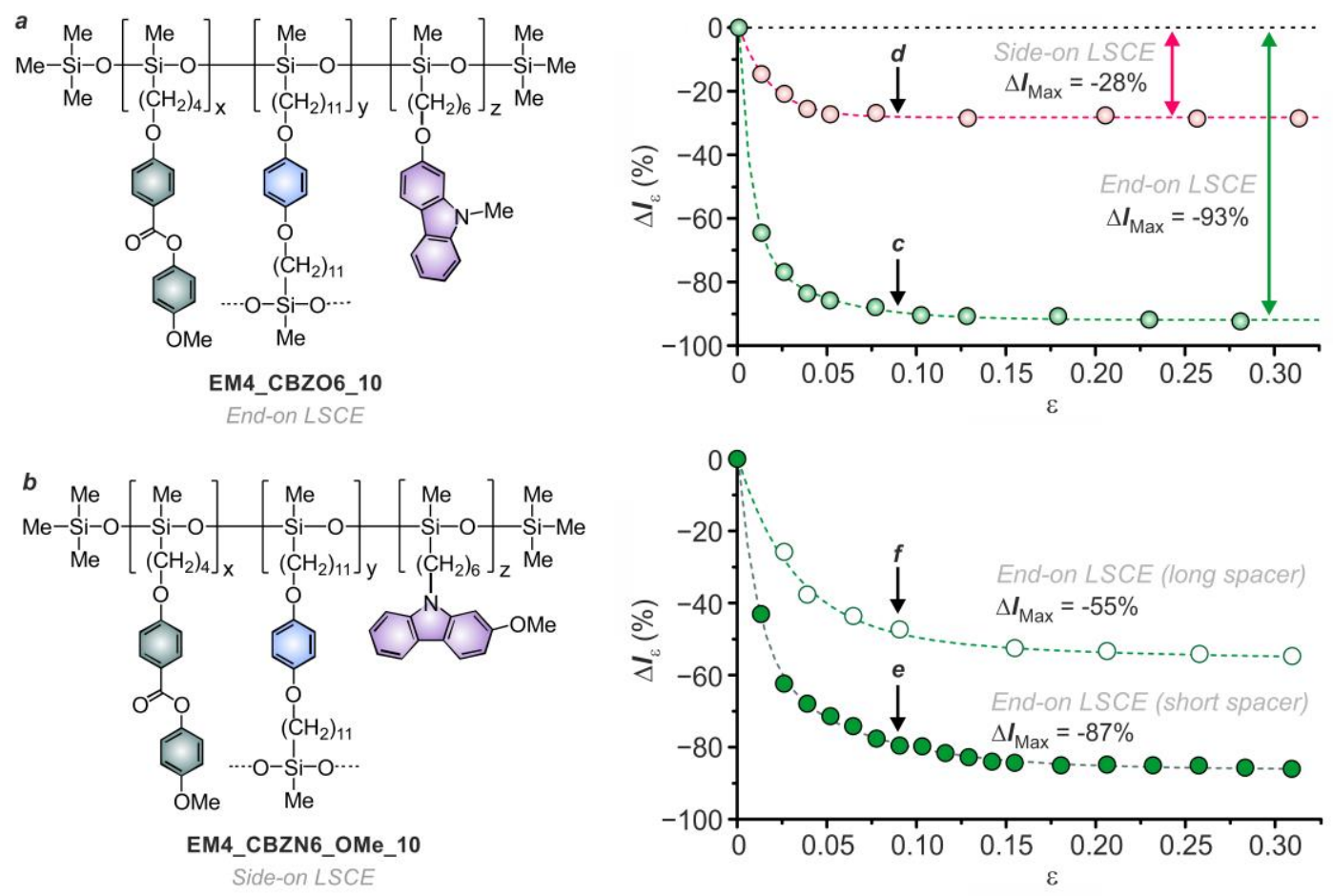

Figure 5. Chemical composition $(\mathbf{a}, \mathbf{b})$ and mechanofluorescent response $(\mathbf{c}, \mathbf{d})$ for the end-on $(\mathbf{a}, \mathbf{c})$ and side-on (b,d) LSCEs EM4_CBZO6_10 and EM4_CBZN6_OMe_10. Mechanofluorescent response for end-on elastomers with a different flexible spacer length $(\mathbf{e}, \mathbf{f})$ [58]. (Copyright from ACS Applied Polymer Materials 2019). 
A comparison between the mechanofluorescent response observed for these two elastomers reveals that the system where the carbazole fluorophores are attached end-on (EM4_CBZO6_10) is significantly more efficient $\left(\Delta I_{\mathrm{Max}}=-93 \%\right.$, in Figure $\left.5 \mathrm{c}\right)$ than its side-on counterpart EM4_CBZN6_OMe_10 $\left(\Delta I_{\mathrm{Max}}\right.$ $=-28 \%$, in Figure 5d) [58]. According to this, an end-on connection of the carbazole fluorophores to the polymer chains, the very same as the mesogens, enables a much stronger interaction with the surrounding mesogen molecules upon deformation and leads, in turn, to more efficient optical force sensors. In this way, the mode by which the carbazole fluorophores are connected to the polymer coil is essential to achieve LSCE-based optical force sensors with improved sensing abilities.

It should be also mentioned that, as for side-on LSCEs, the mechanofluorescence exhibited by end-on LSCEs depends also on the length of the flexible alkyl chain connecting the carbazole fluorogenic moieties to the polysiloxane network. In this case, when short or medium spacers are used, the force applied to the elastomeric material is effectively transmitted from the polymer chains to the pendant carbazole units producing, thus, a major decrease in the material luminescence $\left(\Delta I_{\text {Max }}\right.$ values of $-87 \%$ and $-93 \%$ for $n=3$ and $n=6$, respectively, in Figure $5 \mathrm{c}, \mathrm{e})$. As the conformational freedom of the flexible spacer increases $(n=11)$, the linker can dissipate the mechanical input and compromise the efficiency of the resulting optical force sensor $\left(\Delta I_{\operatorname{Max}}=-55 \%\right.$, in Figure $\left.5 f\right)$ [58].

\subsubsection{Type of Mesophase Exhibited by the LSCE}

Besides connecting the carbazole fluorophores to the main polymer backbone via an end-on connection, endowing side-on elastomeric materials with a different mesogenic arrangement leads also to the conception of very efficient optical force sensors [59]. In this section, four smectic liquid single crystal elastomers will be considered, namely, EM10_CBZN2_10, EM10_CBZN6_10, EM10_CBZN10_10 and EM10_CBZN11_10 (in Figure 6a). In this case, 4-methoxyphenyl 4-(9-decenyloxy)-benzoate (M10) has been used as the mesogenic monomer. Although this low molar mass liquid crystal shows a nematic mesophase within a very narrow temperature range, it leads to stable hexatic smectic $B$ ( $\mathrm{SmB}$, mesogens spacing $\approx 4.3 \AA$, layers spacing $\approx 31 \AA$ ) elastomeric materials at room temperature when incorporated into polysiloxane networks. Specifically, these elastomeric systems also display a smectic A mesophase (SmA, mesogens spacing $\approx 4.3 \AA$, layers spacing $\approx 31 \AA$ ) at higher temperatures (between ca. 306 and $363 \mathrm{~K})$.
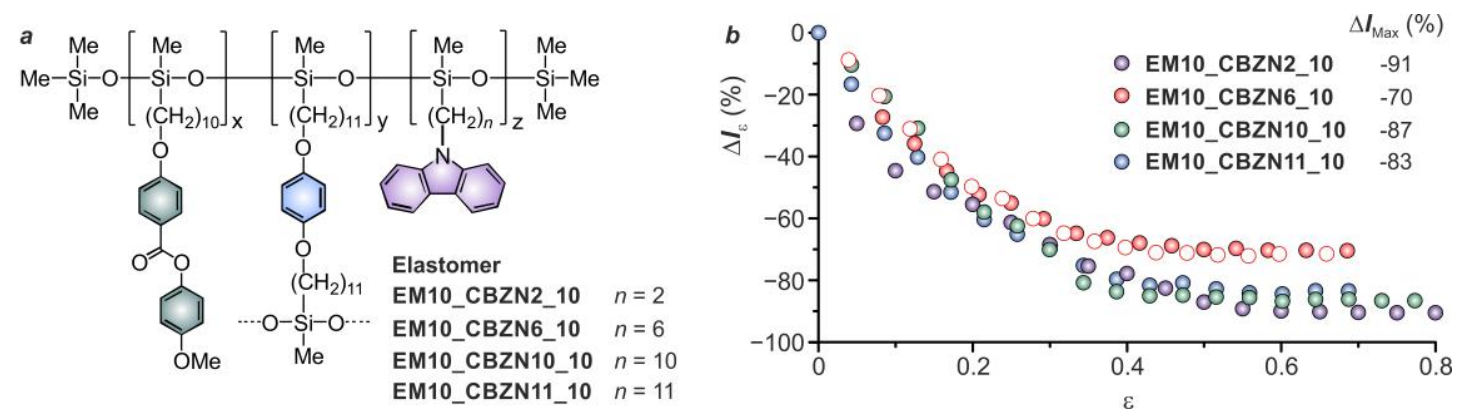

Figure 6. Chemical composition (a) and mechanofluorescent response (b) for the smectic LSCEs EM10_CBZN2_10, EM10_CBZN6_10,EM10_CBZN10_10 and EM10_CBZN11_10. The red empty circles indicate the mechanofluorescent response of elastomer EM10_CBZN10_10 at 328 K, i.e., within the thermal range of stability of its smectic A mesophase [59]. (Copyright from Wiley-VCH Verlag GmbH \& Co. KGaA 2018).

As a main difference with the nematic LSCEs that have been discussed previously in this review, smectic $B$ elastomers show a mechanoluminescent response that is practically independent of the length of the carbazole flexible spacer $\left(\Delta I_{\mathrm{Max}}\right.$ around $-85 \%$, in Figure $\left.6 \mathrm{~b}\right)$ [59]. In addition, it should be remarked that the $\Delta I_{\mathrm{Max}}$ value of the best performing smectic B LSCE (EM10_CBZ_N2_10, $\Delta I_{\mathrm{Max}}$ $=-91 \%)$ is almost threefold higher than the one obtained for the best nematic counterpart $\left(\Delta I_{\operatorname{Max}}=\right.$ $-34 \%$, see Figures 3 and 4). The mechanofluorescent behavior of elastomer EM10_CBZ_N6_10 has also 
been investigated within the thermal range of stability of its SmA mesophase, thereby obtaining the very same $\Delta I_{\text {Max }}$ value than the one obtained under ambient conditions (-72\% vs. $\left.-70 \%\right)$.

These results reveal that the characteristic stratified nature of smectic phases is a main structural factor controlling the efficiency of LSCE-based force sensors. In the case of carbazole-containing smectic LSCEs, the carbazole fluorophores are located within the lamellar structure of the host mesophase, forcing a stronger interaction between both platforms than the one occurring in the case of their nematic analogs. Indeed, this is also the reason by which the observed mechano-fluorescent response is independent of the length of the flexible spacer used to connect the carbazole fluorophores to the main polymeric backbone.

\subsubsection{Performance of the LSCE-Based Optical Force Sensors}

The potential applicability of the LSCE-based optical force sensors has been evaluated by means of time-resolved mechanofluorescence experiments, which involve registering the evolution of the material fluorescence intensity at $\lambda_{\mathrm{Em}}$ over time before and after the application of an external force, in a periodic fashion. Figure 7 shows a representative time-resolved mechanofluorescence experiment for LSCE EM4_CBZN6_10. As can be seen in the figure, the fluorescence intensity of the LSCE can be rapidly and reversibly modulated mechanically. Indeed, characteristic times $\left(\tau_{\mathrm{ON}}\right.$ and $\tau_{\mathrm{OFF}}$ in Figure 7$)$ of $500 \mathrm{~ms}$ have been determined for the decrease in intensity upon stretching of the sample and for the subsequent recovery of the prestress value upon releasing the force, respectively [19]. Note that these characteristic times correspond to the time required to undergo any of these two processes in an extension of $50 \%$. Therefore, carbazole-containing LSCEs are valuable systems to rapidly detect mechanical forces under ambient conditions with simple fluorescence measurements.
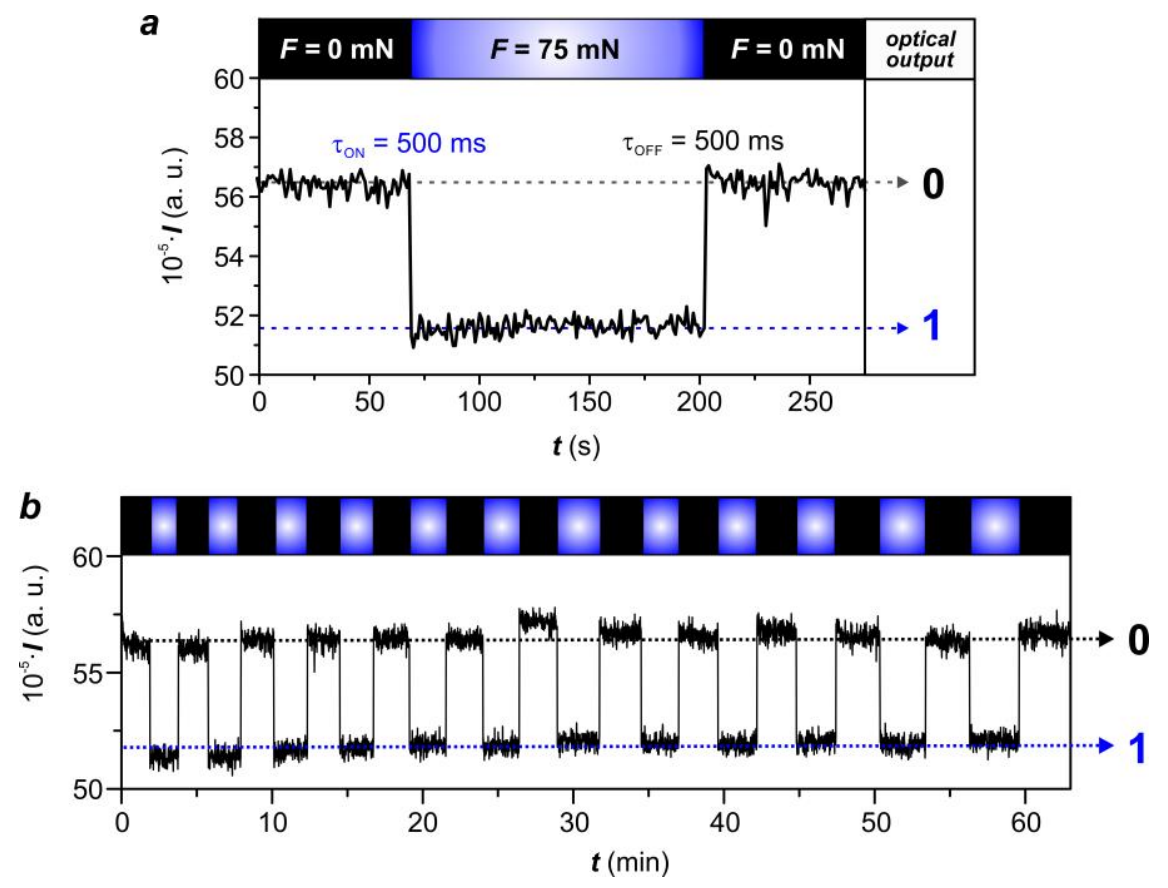

Figure 7. Time-resolved mechanofluorescence experiment for LSCE EM4_CBZN6_10 [19]. (Copyright from Wiley-VCH Verlag GmbH \& Co. KGaA 2015).

\section{Conclusions and Perspectives}

The elongation of carbazole-containing liquid single crystal elastomers, in the direction parallel to their longest axis, provokes a huge decrease in their emission intensity. When the elastomeric material is deformed along its anisotropic direction, i.e., parallel to the director, an increase in its intrinsic nematic 
ordering forces a closer interaction between the carbazole fluorophores and the mesogenic molecules. These differential interactions established at the nanoscale result in a quenching of the luminescence of the formers at the macroscopic level. In this way, two interconvertible states are generated mechanically for the system with excellent contrast, in other words, the luminescence of carbazole-containing LSCEs can be switched from on to off upon deformation. Therefore, carbazole-based LSCEs are valuable fluorescent flexible materials able to convert mechanical sensations into processable optical signals and, thus, they find great applicability as optical force sensors. This review presents to the reader the recent progress made in the design of these emerging materials, emphasizing the different structural factors that play a key role in their performance.

Regarding the carbazole fluorophores, a higher concentration of the organic emitter into the elastomeric network leads to more efficient optical force sensors. Nevertheless, the presence of long range orientational order in the elastomeric material is essential to achieve optical force sensors with these macromolecular networks. The linkage (end-on or side-on) of the carbazole monomers to the elastomeric network also impacts significantly the sensing abilities of the resulting material, with the end-on attachment the one producing the most efficient sensors. In addition, the length of the alkyl chain that binds the organic fluorophores to the polymeric backbone fine-tunes the material performance. In the case of side-on systems, maximum efficiency is achieved when both fluorophores and mesogens have flexible spacers of a comparable length. When end-on fluorophores are considered instead, short spacers (up to 6 methylene units) are preferable since they transmit more efficiently the mechanical input from the polymer chains to the fluorogenic components. Finally, SmB LSCEs, with a layered structure, yield also very efficient optical force sensors independently of the length of the fluorophore flexible spacer. In SmB LSCEs, the fluorogenic platforms and mesogenic units are restricted within the smectic lamellae. Such a feature induces an effective coupling between the fluorogenic and mesogenic components, leading to more efficient optical force sensors than with their nematic analogs. On the basis of these considerations, mechanoluminescent decay is not only controlled by the molecular architecture of the carbazole platform and its connection to the polymeric backbone, but also by the inherent organized nature of the LSCE host mesophase. Importantly, the design schemes summarized in this review have evolved into a new family of efficient fluorescence-based force sensors capable of converting mechanical inputs into optical outputs under ambient conditions. Thus, these findings have undeniable implications in the future design of novel LSCE-based materials for transducing and sensing purposes.

Further investigation in the field might involve the incorporation of side-on or end-on carbazole fluorophores into main-chain nematic or smectic LSCEs, where an even stronger transmission of the mechanical input from the polymeric backbone to the mesogenic and fluorogenic components should be expected. The design of force sensing elastomeric materials that emit light in the form of fluorescence within the visible region of the electromagnetic spectrum would also be an important step forward. Indeed, these novel materials would enable a naked eye visualization of the effect of the applied mechanical input on the elastomer. Finally, the incorporation of non-fluorogenic monomers into the LSCE, which are sensitive to orthogonal external stimuli, such as temperature, light, magnetic fields, etc., might evolve into a second generation of multifunctional optical force sensors with new abilities and enhanced performances.

Author Contributions: Conceptualization, J.G.-A. and D.V.; methodology, J.G.-A. and D.V.; validation, J.G.-A. and D.V.; formal analysis, J.G.-A. and D.V.; investigation, J.G.-A.; resources, D.V.; writing-original draft preparation, J.G.-A. and D.V.; writing-review and editing, J.G.-A. and D.V.; visualization, J.G.-A. and D.V.; supervision, J.G.-A. and D.V.; project administration, J.G.-A. and D.V.; funding acquisition, D.V. All authors have read and agreed to the published version of the manuscript.

Funding: This research was funded by the Ministerio de Ciencia, Innovación y Universidades (Spain)/Agencia Estatal de Investigación (Spain)/Fondo Europeo de Dearrollo Regional (FEDER, European Union), grant number PGC2018-095477-B-I00.

Conflicts of Interest: The authors declare no conflict of interest. 


\section{References}

1. Warner, M.; Terentjev, E.M. Liquid Crystal Elastomers; Clarendon Press: Oxford, UK, 2007.

2. Demus, D.; Goodby, J.W.; Gray, G.W.; Spiess, H.W.; Vill, V. Handbook of Liquid Crystals; Wiley-VCH: Weinheim, Germany, 1998.

3. Fleischmann, E.K.; Zentel, R. Liquid-Crystalline Ordering as a Concept in Materials Science: From Semiconductors to Stimuli-Responsive Devices. Angew. Chem. Int. Ed. 2013, 52, 8810-8827. [CrossRef]

4. Finkelmann, H.; Nishikawa, E.; Pereira, G.G.; Warner, M. A New Opto-Mechanical Effect in Solids. Phys. Rev. Lett. 2001, 87, 015501-1-015501-4. [CrossRef]

5. Hogan, P.M.; Tajbakhsh, A.R.; Terentjev, E.M. UV Manipulation of Order and Macroscopic Shape in Nematic Elastomers. Phys. Rev. E 2002, 65, 0411720. [CrossRef] [PubMed]

6. Broer, D.J.; Crawford, G.P.; Zumer, S. The Liquid Crystals Book Series. Cross-Linked Liquid Crystalline Systems: From Rigid Polymer Networks to Elastomers; CRC Press: Boca Raton, FL, USA, 2011.

7. Camacho-López, M.; Finkelmann, H.; Palffy-Muhoray, P.; Shelley, M. Fast Liquid-Crystal Elastomer Swims into the Dark. Nat. Mater. 2004, 3, 307-310. [CrossRef] [PubMed]

8. Garcia-Amorós, J.; Finkelmann, H.; Velasco, D. Influence of the Photo-Active Azo Cross-Linker Spacer on the Opto-Mechanics of Polysiloxane Elastomer Actuators. J. Mater. Chem. 2011, 21, 1094-1101. [CrossRef]

9. Garcia-Amorós, J.; Piñol, A.; Finkelmann, H.; Velasco, D. Azophenol-Based Liquid-Crystalline Elastomers for Light-Driven Actuators. Org. Lett. 2011, 13, 2282-2285. [CrossRef] [PubMed]

10. Garcia-Amorós, J.; Martínez, M.; Finkelmann, H.; Velasco, D. Photoactuation and Thermal Isomerisation Mechanism of Cyanoazobenzene-Based Liquid Crystal Elastomers. Phys. Chem. Chem. Phys. 2014, 16, 8448-8454. [CrossRef]

11. Chi, Z.; Zhang, X.; Xu, B.; Zhou, X.; Ma, C.; Zhang, Y.; Liu, S.; Xu, J. Recent Advances in Organic Mechanofluorochromic Materials. Chem. Soc. Rev. 2012, 41, 3878-3896. [CrossRef]

12. Ma, Z.; Wang, Z.; Teng, M.; Xu, Z.; Jia, X. Mechanically Induced Multicolor Change of Luminescent Materials. ChemPhysChem 2015, 16, 1811-1828. [CrossRef]

13. Xu, J.; Chi, Z. (Eds.) Mechanochromic Fluorescent Materials: Phenomena, Materials and Applications; RSC Smart Materials Series, No. 8; Royal Society of Chemistry: Cambridge, UK, 2014.

14. Dong, Y.Q.; Lam, J.W.Y.; Tang, B.Z. Mechanochromic Luminescence of Aggregation-Induced Emission Luminogens. J. Phys. Chem. Lett. 2015, 6, 3429-3436. [CrossRef]

15. Chen, J.; Tang, B.Z. Aggregation-induced Emission: Fundamentals and Applications; Qin, A., Tang, B.Z., Eds.; Wiley-VCH: Chichester, UK, 2014; Volume 1, pp. 307-322.

16. Huang, X.; Qian, L.; Zhou, Y.; Liu, M.; Cheng, Y.; Wu, H. Effective Structural Modification of Traditional Fluorophores to Obtain Organic Mechanofluorochromic Molecules. J. Mater. Chem. C 2018, 6, 5075-5096. [CrossRef]

17. Di, B.-H.; Chen, Y.-L. Recent Progress in Organic Mechanoluminescent Materials. Chin. Chem. Lett. 2018, 29, 245-251. [CrossRef]

18. Jin, M.; Seki, T.; Ito, H. Mechano-Responsive Luminescence via Crystal-to-Crystal Phase Transitions between Chiral and Non-Chiral Space Groups. J. Am. Chem. Soc. 2017, 139, 7452-7455. [CrossRef]

19. Garcia-Amorós, J.; Velasco, D. Optical mechanotransduction with carbazole-based liquid single-crystal elastomers. Macromol. Rapid Commun. 2015, 36, 755-761. [CrossRef]

20. Wei, R.B.; He, Y.N.; Wang, X.G.; Keller, P. Photoluminescent Nematic Liquid Crystalline Elastomer with a Thermomechanical Emission Variation Function. Macromol. Rapid Commun. 2014, 35, 1571-1577. [CrossRef]

21. Wei, R.B.; Zhang, H.X.; He, Y.N.; Wang, X.G.; Keller, P. Photoluminescent Nematic Liquid Crystalline Elastomer Actuators. Liq. Cryst. 2014, 41, 1821-1830. [CrossRef]

22. Davis, D.A.; Hamilton, A.; Yang, J.; Cremar, L.D.; Van Gough, D.; Potisek, S.L.; Ong, M.T.; Braun, P.V.; Martínez, T.J.; White, S.R.; et al. Force-Induced Activation of Covalent Bonds in Mechanoresponsive Polymeric Materials. Nature 2009, 459, 68-72. [CrossRef]

23. Kingsbury, C.M.; May, P.A.; Davis, D.A.; White, S.R.; Moore, J.S.; Sottos, N.R. Shear Activation of Mechanophore-Crosslinked Polymers. J. Mater. Chem. 2011, 21, 8381-8388. [CrossRef]

24. Kim, T.A.; Robb, M.J.; Moore, J.S.; White, S.R.; Sottos, N.R. Mechanical Reactivity of Two Different Spiropyran Mechanophores in Polydimethylsiloxane. Macromolecules 2018, 51, 9177-9183. [CrossRef] 
25. Imato, K.; Kanehara, T.; Ohishi, T.; Nishihara, M.; Yajima, H.; Ito, M.; Takahara, A.; Otsuka, H. Mechanochromic Dynamic Covalent Elastomers: Quantitative Stress Evaluation and Autonomous Recovery. ACS Macro Lett. 2015, 4, 1307-1311. [CrossRef]

26. Ducrot, E.; Chen, Y.; Bulters, M.; Sijbesma, R.P.; Creton, C. Toughening Elastomers with Sacrificial Bonds and Watching them Break. Science 2014, 344, 186-189. [CrossRef]

27. Crenshaw, B.R.; Weder, C.J. Deformation Induced Color Changes in Melt-Processed Photoluminescent Polymer Blends. Chem. Mater. 2003, 15, 4717-4724. [CrossRef]

28. Weder, C.J. Mechanoresponsive Materials. Mater. Chem. 2011, 21, 8235-8236. [CrossRef]

29. Calvino, C.; Neumann, L.; Weder, C.; Schrettl, S. Approaches to Polymeric Mechanochromic Materials. J. Polym. Sci. Pol. Chem. 2017, 55, 640-652. [CrossRef]

30. Muramatsu, T.; Sagara, Y.; Traeger, H.; Tamaoki, N.; Weder, C. Mechanoresponsive Behavior of a PolymerEmbedded Red-Light Emitting Rotaxane Mechanophore. ACS Appl. Mater. Interfaces 2019, 11, 24571-24576. [CrossRef]

31. Sagara, Y.; Karman, M.; Seki, A.; Pannipara, M.; Tamaoki, N.; Weder, C. Rotaxane-Based Mechanophores Enable Polymers with Mechanically Switchable White Photoluminescence. ACS Cent. Sci. 2019, 5, 874-881. [CrossRef]

32. Sulkanen, A.R.; Sung, J.; Robb, M.J.; Moore, J.S.; Sottos, N.R.; Liu, G.Y. Spatially Selective and DensityControlled Activation of Interfacial Mechanophores. J. Am. Chem. Soc. 2019, 141, 4080-4085. [CrossRef]

33. Zhao, D.; Li, G.; Wu, D.; Qin, X.; Neuhaus, P.; Cheng, Y.; Yang, S.; Lu, Z.; Pu, X.; Long, C.; et al. Regiospecific $\mathrm{N}$ Heteroarylation of Amidines for Full Color Tunable Boron Difluoride Dyes with Mechanochromic Luminescence. Angew. Chem. Int. Ed. 2013, 52, 13676-13680. [CrossRef]

34. Li, G.; Song, F.; Wu, D.; Lan, J.; Liu, X.; Wu, J.; Yang, S.; Xiao, D.; You, J. Cation-Anion Interaction Directed Molecular Design Strategy for Mechanochromic Luminescence. Adv. Funct. Mater. 2014, 24, 747-753. [CrossRef]

35. Zhang, Z.; Xue, P.; Gong, P.; Zhang, G.; Peng, J.; Lu, R. Mechanofluorochromic Behaviors of Biminoenolate Boron Complexes Functionalized with Carbazole. J. Mater. Chem. C 2014, 2, 9543-9551. [CrossRef]

36. Tanioka, M.; Kamino, S.; Muranaka, A.; Ooyama, Y.; Ota, H.; Shirasaki, Y.; Horigome, J.; Ueda, M.; Uchiyama, M.; Sawada, D.; et al. Reversible Near-Infrared/Blue Mechanofluorochromism of Aminobenzopyranoxanthene. J. Am. Chem. Soc. 2015, 137, 6436-6439. [CrossRef]

37. Matsunaga, Y.; Yang, J.-S. Multicolor Fluorescence Writing Based on Host-Guest Interactions and Force Induced Fluorescence Color Memory. Angew. Chem. Int. Ed. 2015, 54, 7985-7989. [CrossRef]

38. Jiang, Y.; Gindre, D.; Allain, M.; Liu, P.; Cabanetos, C.; Roncali, J. A Mechanofluorochromic Push-Pull Small Molecule with Aggregation Controlled Linear and Nonlinear Optical Properties. Adv. Mater. 2015, 27, 4285-4289. [CrossRef]

39. Yoshii, R.; Suenaga, K.; Tanaka, K.; Chujo, Y. Mechanofluorochromic Materials Based on Aggregation Induced Emission Active Boron Ketoiminates: Regulation of the Direction of the Emission Color Changes. Chem. Eur. J. 2015, 21, 7231-7237. [CrossRef]

40. Nishida, T.; Ohta, S.; Xu, F.; Shinohara, K.; Kamada, T.; Akashi, H.; Takezaki, M.; Wakamatsu, K.; Orita, A. Dual Emission and Mechanofluorochromism of a V-Shaped $\pi$-System Composed of Disulfonyl-Substituted Dibenzocyclooctatetraenes. Org. Lett. 2016, 18, 3988-3991. [CrossRef]

41. Xue, P.; Yao, B.; Liu, X.; Sun, J.; Gong, P.; Zhang, Z.; Qian, C.; Zhang, Y.; Lu, R. Reversible Mechanochromic Luminescence of Phenothiazine-based 10,10'-Bianthracene Derivatives with Different Lengths of Alkyl Chains. J. Mater. Chem. C 2015, 3, 1018-1025. [CrossRef]

42. Zhang, Z.; Wu, Z.; Sun, J.; Yao, B.; Xue, P.; Lu, R. $\beta$-Iminoenolate Boron Complex with Terminal Triphenylamine Exhibiting Polymorphism and Mechanofluorochromism. J. Mater. Chem. C 2016, 4, 2854-2861. [CrossRef]

43. Yang, H.; Ye, K.; Sun, J.; Gong, P.; Lu, R. Mechanofluorochromic Behaviors of Salicylaldimine Difluoroboron Complexes. Asian J. Org. Chem. 2017, 6, 199-206. [CrossRef]

44. Zhao, J.; Chi, Z.; Yang, Z.; Mao, Z.; Zhang, Y.; Ubba, E.; Chi, Z. Recent Progress in the Mechanofluorochromism of Distyrylanthracene Derivatives with Aggregation-Induced Emission. Mater. Chem. Front. 2018, 2, 1595-1608. [CrossRef]

45. Zhao, W.; He, Z.; Peng, Q.; Lam, J.W.Y.; Ma, H.; Qiu, Z.; Chen, Y.; Zhao, Z.; Shuai, Z.; Dong, Y.; et al. Highly Sensitive Switching of Solid-State Luminescence by Controlling Intersystem Crossing. Nat. Commun. 2018, 9 , 3044. [CrossRef] 
46. Sharber, S.A.; Shih, K.-C.; Mann, A.; Frausto, F.; Haas, T.E.; Nieh, M.-P.; Thomas, S.W. Reversible Mechanofluorochromism of Aniline-Terminated Phenylene Ethynylenes. Chem. Sci. 2018, 9, 5415-5426. [CrossRef]

47. Sagara, Y.; Karman, M.; Verde-Sesto, E.; Matsuo, K.; Kim, Y.; Tamaoki, N.; Weder, C. Rotaxanes as Mechanochromic Fluorescent Force Transducers in Polymers. J. Am. Chem. Soc. 2018, 140, 1584-1587. [CrossRef]

48. Calvino, C.; Sagara, Y.; Buclin, V.; Haehnel, A.P.; del Prado, A.; Aeby, C.; Simon, Y.C.; Schrettl, S.; Weder, C. Mechanoresponsive, Luminescent Polymer Blends Based on an Excimer Forming Telechelic Macromolecule. Macromol. Rapid Commun. 2019, 40, 1800705. [CrossRef]

49. Louis, M.; Sethy, R.; Kumar, J.; Katao, S.; Guillot, R.; Nakashima, T.; Allain, C.; Kawai, T.; Métivier, R. Mechano-Responsive Circularly Polarized Luminescence of Organic Solid-State Chiral Emitters. Chem. Sci. 2019, 10, 843-847. [CrossRef]

50. Hirai, Y.; Wrona-Piotrowicz, A.; Zakrzewski, J.; Brosseau, A.; Guillot, R.; Métivier, R.; Allain, C. Mechanofluorochromism of pyrene-derived amidophosphonates. Photochem. Photobiol. Sci. 2020. [CrossRef]

51. Li, Y.-X.; Chen, Y.-G.; Yu, Z.-F.; Yang, X.-F.; Nie, Y.; Cui, Y.; Sun, G.-X. Polymorph-Dependent Multicolor-Switchable Mechanofluorochromism of 1-(9-Anthryl)vinyl-3-(3-pyridyl)vinylbenzene: Molecular Conformation versus Intermolecular Interaction. J. Phys. Chem. C 2020, 124, 3784-3792. [CrossRef]

52. Küpfer, J.; Finkelmann, H. Nematic Liquid Single Crystal Elastomers. Makromol. Chem. Rapid Commun. 1991, 12, 717-726. [CrossRef]

53. Kim, H.; Zhu, B.; Adetiba, O.; Agrawal, A.; Ajayan, P.; Jacot, J.G.; Verduzco, R. Preparation of Monodomain Liquid Crystal Elastomers and Liquid Crystal Elastomers Composites. J. Vis. Exp. 2016, 108, 53688.

54. Lovell, R.; Mitchell, G.R. Molecular Orientation Distribution Derived from an Arbitrary Reflection. Acta Cryst. 1981, A37, 135-137. [CrossRef]

55. Mitchell, G.R.; Windle, A. Orientation in Liquid Crystal Polymers. In Developments in Crystal Polymers-2; Basset, D.C., Ed.; Science: London, UK; New York, NY, USA, 1988; pp. 115-175.

56. Schatzle, J.; Kaufhold, W.; Finkelmann, H. Nematic Elastomers: The Influence of External Mechanical Stress on the Liquid-Crystalline Phase Behavior. Makromol. Chem. 1989, 190, 3269-3284. [CrossRef]

57. Garcia-Amorós, J.; Bassaganyas, S.; Velasco, D. Exploring Optical Mechanotransduction in Fluorescent Liquid Crystal Elastomers. Phys. Chem. Chem. Phys. 2016, 18, 5108-5111. [CrossRef]

58. Heras, D.; Reig, M.; Llorca-Isern, N.; Garcia-Amorós, J.; Velasco, D. Highly Efficient Elastomeric Fluorescence Sensors for Force Detection. ACS Appl. Polym. Mater. 2019, 1, 535-541. [CrossRef]

59. Escalera-López, D.; Garcia-Amorós, J.; Velasco, D. Smectic-B Liquid Single Crystal Elastomers as Efficient Optical Mechanotransducers. Macromol. Chem. Phys. 2018, 219, 1700550. [CrossRef] 